\title{
IDENTIFICATION OF MECHANICAL LOAD FOR ElECTRICAL DRIVES COMMISSIONING - LABElling MaChine CASE STUdy
}

\author{
Luca Peretti, Student Member, IEEE, Mauro Zigliotto, Member, IEEE
}

\begin{abstract}
The paper presents a result of the system identification theory applied to an industrial machine. The case study was a labelling machine driven by a permanent magnet synchronous motor drive. The identified system model has been used to tune the closedloop speed control of the drive. A brief recall of the basic principles of power spectral density applied to model identification, hints for the practical implementation and experimental results are included in the paper.
\end{abstract}

Index Terms: Drive commissioning, System identification, AC machines.

\section{INTRODUCTION}

Electric drives commissioning often calls for onsite tuning of current and speed controllers. The tuning is performed by a trained employee who adjusts the regulators parameters in order to get the best performances. Several approaches can be adopted. The first and well-known method for PI tuning is the Ziegler-Nichols method [1], [2], which tunes the parameters by means of a heuristic formula. The method, anyway, often requires manual refinements to obtain satisfactory performances.

A more recent trend is represented by selfcommissioning. The literature presents several examples of self-commissioning procedures for PI regulators tuning. A fair comparison of identification techniques is reported in [3]. Self-tuning algorithms yield faster commissioning stages, as well as cost reductions, since the procedures can be easily implemented in the drive and executed once at drive set-up. Moreover, precise self-tuning algorithms can theoretically consider more complex models and solve more complicated expressions for parameters calculation, which reflects in higher performances with respect to drives tuned manually. An interesting solution has been presented in [4], where the load is identified assuming a two-mass system structure with dominant elasticity. Results of the two-mass system model commissioning can be also used to propose different control concepts, as reported in [5], where a knowledge-based controller selector based on fuzzy rules returns a rating of control parameters, based on their theoretical feasibility with respect to the identified model.

Another solution for self-tuning of the speed PI regulator is reported in [6], where the approach is based on the information retrieved by forcing the system to oscillate at predetermined frequencies. The permanent oscillations are induced by non-linear blocks (relay with hysteresis) introduced on purpose inside the control loop, and then removed once the parameters of the regulators have been calculated.
However, in many cases the mechanical load is either unknown a-priori or very complex, while its full identification would be of great help in the design of the control system. In principle, a mathematical approach based on theoretical modelling could be possible, provided that nameplate data of every mechanical component is present. However, this approach goes rapidly off-limits, as the mechanical structure complexity increases. In such cases, nonparametric off-line approaches should be preferred, as the load is identified recording its excitation (speed) as function of an injected perturbation (torque), and by post-processing the results.

A straightforward non-parametric approach to the estimation of the mechanical load transfer function is the addition of different sinusoidal torque components to a constant torque reference, recording the sinusoidal speed variation in output after the end of each initial transient. By measuring both amplitude and phase of the speed variation with respect to the torque sinusoidal contribute, and repeating the procedure for different frequencies, the transfer function of the mechanical load can be inferred.

Actually, the approach hides several drawbacks. The variation of the frequency is time-consuming, especially when the transfer function must be carefully identified around critical resonance points. The operator is asked to validate the results while the procedure is executed, not to lose relevant information about the load.

On the other hand, solutions like [7] and [8] seem attractive but of rather complex fruition in industrial applications. Effective solutions exploit different types of signal perturbation, whose frequency spectrum is wider than that of a simple sinusoid. In this way, the load identification is faster since the operator is not requested to manually vary the injected frequency. However, the frequency interval and the harmonic magnitude profile are two of the most important parameters for the choice of a suitable disturbance signal.

A fair example is reported in [4], where the perturbation is represented by a pseudo-random pattern injected as a disturbance signal into the torque reference of a speed-and-current-controlled drive. Differently from the manual approach, the acquisition of the magnitude and phase lag of every harmonic with respect to the injected perturbation is not feasible, due to the wide frequency content of both input and output signals. The load identification is thus obtained by a power spectral density approach, which has also been used in this work, with a peculiar signal injection pattern.

This paper presents an off-line identification 
procedure based on the power density approach applied to a labelling machine, whose complex mechanical load transfer function was completely unknown. The identification has been performed at different load conditions, which cover its safe operating range.

The identified model has been exploited to complete the commissioning of the speed loop of the PMSM drive of the machine.

An added value of the work is that the procedure is simple enough to be easily implemented on any test-bench, or even in the hardware of the machine. It can be obviously extended to several different industrial machines, and the labelling machines here presented can be just intended as a successful case study. Experimental results of both the identification procedure and the speed-controlled system are presented, confirming the validity of the approach.

\section{TRANSFER FUNCTION IDENTIFICATION}

\section{A. The power spectral density}

The power spectral density approach, which is exploited for the transfer function estimation in this work, is based on the stochastic processes theory [9]. The power spectral density can be defined for stationary processes only, which have a time-invariant statistical description. In particular, a stationary process features a constant statistical mean:

$$
\begin{aligned}
m_{x}(t) & =E[x(t)]=\int_{-\infty}^{+\infty} a f_{x}(a, t) d a \\
& =\int_{-\infty}^{+\infty} a f_{x}(a, 0) d a=m_{x}
\end{aligned}
$$

where $f_{x}(a, t)$ is the probability density function of $x(t)$ to assume the value $a$. It is demonstrated that the autocorrelation of a stationary process, defined as

$$
r_{x}(t, t+\tau)=E[x(t+\tau) x(t)]
$$

depends only on the difference $t$ - $\tau$ and it is a positive semi-definite function, since the following properties occur:

$$
\begin{aligned}
& r_{x}(0)=E\left|x^{2}(t)\right| \geq 0 \\
& r_{x}(\tau)=r_{x}(-\tau) \\
& r_{x}(0) \geq\left|r_{x}(\tau)\right| \quad \forall \tau
\end{aligned}
$$

The Fourier transformation of the autocorrelation function is the power spectral density, defined as

$$
R_{x}(f)=\int_{-\infty}^{+\infty} r_{x}(\tau) e^{-j 2 \pi f \tau} d \tau
$$

for continuous-time processes. $R_{x}(f)$ is greater or equal to zero because $r_{x}(t)$ is positive semi-definite. A mutual power spectral density can be defined between two stationary processes $x(t)$ and $y(t)$ :

$$
\begin{aligned}
R_{y x}(f) & =\int_{-\infty}^{+\infty} r_{y x}(\tau) e^{-j 2 \pi f \tau} d \tau \\
& =\int_{-\infty}^{+\infty} E[y(t+\tau) x(t)] e^{-j 2 \pi f \tau} d \tau
\end{aligned}
$$

\section{B. The transfer function identification}

The hypothesis is that input and output processes $x(t)$ and $y(t)$ are linked by a linear transformation. Realisations of both processes are available, while the transfer function is unknown and it is going to be estimated by the power spectral density approach. The mathematical relationship between $y(t)$ and $x(t)$ is the following:

$$
y(t)=\int_{-\infty}^{+\infty} x(\tau) h(t-\tau) d \tau
$$

Where $h(t)$ is the impulsive response of the system. If $x(t)$ is a stationary process, the expected value of $y(t)$ is expressed by:

$$
\begin{aligned}
E[y(t)] & =E\left[\int_{-\infty}^{+\infty} x(\tau) h(t-\tau) d \tau\right] \\
& =\int_{-\infty}^{+\infty} E[x(\tau)] h(t-\tau) d \tau \\
& =m_{x} \int_{-\infty}^{+\infty} h(\tau) d \tau=m_{x} H(0)
\end{aligned}
$$

where $m_{x}$ is the expected value of $x(t)$ and $H(0)$ is the Fourier transform calculated for the zero frequency of $h(t)$. The correlation between $y(t)$ and $x(t)$ is equal to:

$$
\begin{aligned}
r_{y x}(t, t+\tau)= & E[y(t+\tau) x(t)] \\
= & E\left[\int_{-\infty}^{+\infty} x\left(t+\tau-t_{1}\right) h\left(t_{1}\right) d t_{1} \cdot x(t)\right] \\
= & E\left[\int_{-\infty}^{+\infty} x\left(t+\tau-t_{1}\right) x(t) h\left(t_{1}\right) d t_{1}\right] \\
& =\int_{-\infty}^{+\infty} E\left[x\left(t+\tau-t_{1}\right) x(t)\right] h\left(t_{1}\right) d t_{1} \\
& +\infty \\
= & \int_{-\infty}^{+\infty} r_{x}\left(\tau-t_{1}\right) h\left(t_{1}\right) d t_{1}=h * r_{x}(\tau)
\end{aligned}
$$


where $r_{x}(\tau)$ is the autocorrelation of $x(t)$ and the symbol $*$ is the convolution operator. From (7) and (8), it follows that $y(t)$ is a stationary process. With similar calculations, it can be obtained that:

$$
\begin{aligned}
& r_{x y}(\tau)=h(-t) * r_{x}(\tau) \\
& r_{y}(\tau)=h(-t) * h(t) * r_{x}(\tau)
\end{aligned}
$$

Calculating the Fourier transformation of both the expressions in (9), and recalling that a convolution in the time domain corresponds to a multiplication in the frequency domain (and vice versa), it is

$$
\begin{aligned}
& R_{y x}(f)=H(f) R_{x}(f) \\
& R_{x y}(f)=H^{*}(f) R_{x}(f) \\
& R_{y}(f)=|H(f)|^{2} R_{x}(f)
\end{aligned}
$$

where $H^{*}(f)$ is the complex conjugate transfer function. The transfer function can be estimated from the first of (10), by the knowledge of the spectral densities $R_{y x}(f)$ and $R_{x}(f)$. Incidentally, the spectral densities calculation needs the probability density function, which is usually not known for both the input and the output.

The problem is overcome by using the periodogram method, which refers to ergodic stochastic processes (as are the processes usually involved). For an ergodic process, the arithmetic mean of a single realisation of the process tends to the statistical mean if the temporal window of the realisation is large enough [11]:

$$
m_{x}=E[x(t)]=\lim _{T \rightarrow+\infty} \frac{1}{T} \int_{-T / 2}^{T / 2} x(t) d t
$$

In this case, the Fourier transformation of the process can be approximated by the average of the Fourier transformation of $M$ realisations of the process itself. It can be demonstrated that the spectral densities are approximated by:

$$
\begin{aligned}
& R_{x}(f) \cong \frac{1}{T M} \sum_{i=1}^{M} X_{T_{i}}(f) X_{T_{i}}^{*}(f) \\
& R_{y x}(f) \cong \frac{1}{T M} \sum_{i=1}^{M} Y_{T_{i}}(f) X_{T_{i}}^{*}(f)
\end{aligned}
$$

where $X_{T}(f)$ and $X_{T}(f)$ are the Fourier transformations of $x(t)$ and $y(t)$ realizations in the temporal window $T$.

\section{THE PROPOSED APPROACH}

According to the results expressed in (10) and (12), the transfer function of a generic mechanical load connected to an electrical drive can be estimated by means of the power spectral density approach. The realisations of the input and output processes $x(t)$ and $y(t)$ are represented by the measurements of the torque producing component $i_{q}$ of the stator current and the mechanical speed $\omega_{m}$, respectively.

Fig. 1 shows the block schematic that has been used to identify the mechanical load of a labelling machine. The system is composed by a classic cascade control, with a speed PI controller (outer loop) and a current PI controller (inner loop). In this way, the system is stable and controlled, while the off-line identification process is executed. A test signal is added to the current reference $i_{q}{ }^{*}$. The reference is reproduced by the inner control loop, and a torque disturbance appears to the input of $G M$, which represents the mechanical load. It is worth to note that the spectral characteristics of the injected signal are fundamental for a proper estimation of the mechanical load transfer function, as it is pointed out later on in this Section.

The acquired input and output process realisations are the current $i_{q}$ and the mechanical speed $\omega_{m}$.

During the load identification, the current loop should be tuned for best performances, in order to track the reference (and the injected perturbation) as close as possible. On the contrary, a perfectly tuned speed regulator would lead to a marked speed disturbances rejection, neutralising the effects on the speed of the injected perturbation. Consequently, the speed PI controller should be tuned for a rather low

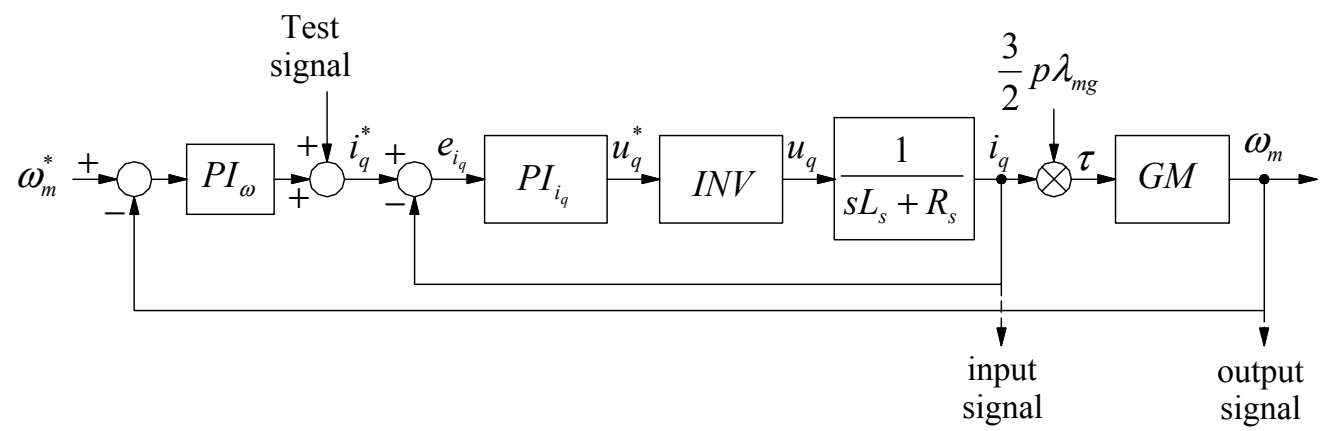

Fig. 1 - Block scheme of the identification process. 
bandwidth. This assures significant speed variations during the torque perturbation and a high signal-tonoise ratio for the identification process.

Spectral characteristics of the injected signal are fundamental for the transfer function identification in the frequency range of interest. To this aim, the chirp signal is a good candidate that combines an easy experimental implementation with effectiveness of the results. In general, a chirp is a sinusoidal signal with a time-variant frequency. It can be described by the following expression:

$$
c(t)=\sin \left(2 \pi \int_{0}^{t} f\left(t^{\prime}\right) d t^{\prime}\right)
$$

If the frequency variation is linear, also the chirp signal is named linear:

$$
f(t)=f_{0}+k t \Rightarrow c(t)=\sin \left(2 \pi\left(f_{0}+\frac{k}{2} t\right) t\right)
$$

Fig. 2 reports an example of chirp signal with linearly-changing frequency between $0 \mathrm{~Hz}$ and $10 \mathrm{~Hz}$. The main advantage of the chirp signal is that mechanical harmonics are of constant amplitude and they do not require excessive peak power to be induced on a common drive. A good choice of the chirp amplitude leads to a relevant signal-to-noise ratio with respect to signals in which frequency components have different amplitudes, as it happens in step-like signals.

It is demonstrated [10] that the product of the chirp frequency variation $\Delta f$ by the temporal window $T$, named time-bandwidth product, has to be large enough to approximate the chirp power spectral density over the whole $\Delta f$.

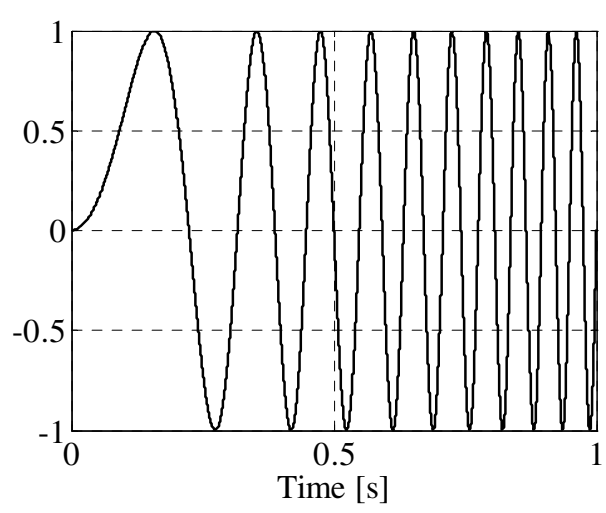

Fig. 2 - Chirp signal between $0 \mathrm{~Hz}$ and $10 \mathrm{~Hz}$ in $1 \mathrm{~s}$.

\section{IV.EXPERIMENTAL RESULTS}

\section{A. The experimental test-bench}

A picture of the experimental setup used for the identification process is shown in Fig. 3.

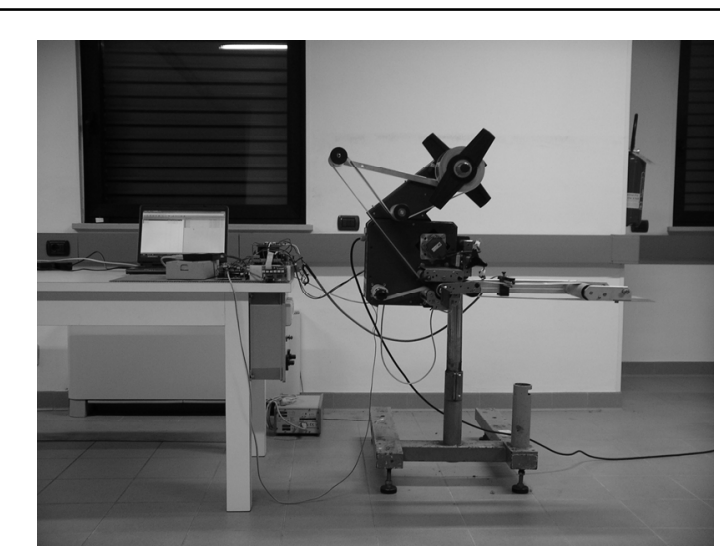

Fig.3 - The experimental setup.

At laboratory premises, the PMSM mounted in the labelling machine was fed by a standard PWM inverter. PMSM's nameplate data are reported in Table 1. For the sake of a rapid time-to-market research, a fast control prototyping (FCP) system was used to implement the identification mechanism and for feeding the PWM inverter by the appropriate test signals. Anyway, the same procedure shall be embedded in the labelling machine firmware.

Rotor position was measured by a conventional encoder, post-processed by the FCP system. A cascade speed-and-current PI control has been implemented on board, as shown in the scheme of Fig. 2. Current controllers have been experimentally tuned by means of a step variation of the phase

Table 1. PMSM parameters

\begin{tabular}{|c|c|}
\hline Parameter & Value \\
\hline Nominal power & $400 \mathrm{~W}$ \\
\hline Nominal speed & $3000 \mathrm{rpm}$ \\
\hline Nominal torque & $1,3 \mathrm{Nm}$ \\
\hline Nominal current & $2,6 \mathrm{Arms}$ \\
\hline Pole pairs & 4 \\
\hline Stator resistance & $1,9 \Omega$ \\
\hline Stator inductance & $10,2 \mathrm{mH}$ \\
\hline Flux due to magnets & $0,059 \mathrm{Vs} / \mathrm{rad}$ \\
\hline
\end{tabular}

current. A bandwidth of around $1 \mathrm{kHz}$ has been obtained for the inner current control loop.

As regards the tuning of the external speed controller, it has been already mentioned that a perfectly tuned PI regulator collides with the need of a high signal-to-noise ratio during the identification process. Thus, the identification process has been carried excluding the integral part of the speed PI regulator, while maintaining only the proportional part, which has been tuned for a bandwidth of few Hertz. 


\section{B. Chirp signal acquisition and post-processing}

The acquisition procedure is composed by few steps, as reported hereafter:

- the motor rotates at one-third of the rated speed (1000 rpm) without any signal injection;

- the chirp signal injection in the current reference signal $i_{q}^{*}$ is activated;

- the actual torque-producing current $i_{q}$ and the mechanical speed $\omega_{m}$ are sampled and acquired for a period of 68 seconds, exploiting the full memory depth of the drive.

The frequency span for the chirp signal injection encompasses frequencies from $0,01 \mathrm{~Hz}$ to $700 \mathrm{~Hz}$, in order to identify the mechanical load in the whole range of interest. Frequencies higher than $700 \mathrm{~Hz}$ are not meaningful, since the speed controller bandwidth will not be higher than that value. In any case, the upper limit is imposed by the current controller, which is not capable of tracking torque references with a frequency higher than that of its bandwidth. Moreover, a torque reference signal with higher frequencies results in poor tracking performance and a noisy speed with inherently low information content.

For the sake of accuracy, the whole frequency span has been divided into eight smaller intervals: [0,01 Hz; 0,1 Hz], [0,1 Hz; $1 \mathrm{~Hz}],[1 \mathrm{~Hz} ; 10 \mathrm{~Hz}],[1$ $\mathrm{Hz} ; 7 \mathrm{~Hz}$ ], $[7 \mathrm{~Hz} ; 15 \mathrm{~Hz}$, [15 Hz; $60 \mathrm{~Hz}$ ], [60 Hz; $150 \mathrm{~Hz}$, [150 Hz; $700 \mathrm{~Hz}$. The procedure has been repeated ten times for each interval and results have been averaged.

The mechanical load transfer function has been evaluated in both no-load (without the label roll) and at full-load (with the complete label roll) conditions, in order to investigate the effects of load variability in the model. The theoretical approach of Section II has been used for the data post-processing, obtaining the amplitude-and-phase Bode diagram of Fig. 4 and Fig. 5.

The spikes of Fig. 5 refer to the borders of the evaluated frequency intervals, and should be neglected. The sudden phase swap between $+/-180$ degrees is only a math issue and should be neglected as well.

Results clearly show the mechanical pole of the system, which is due to the inertia and the viscous friction, located around $1 \mathrm{~Hz}$. Different couples of complex poles and zeros appear in the frequency interval $[40 \mathrm{~Hz} ; 200 \mathrm{~Hz}$, with a profile which depends on the load condition. However, the transfer function dependence on the label roll presence is not dramatic at all.

This convenient result leads to more homogeneous performances of the speed PI controller in the whole load range, once the latter has been tuned for the worst condition. The mechanical torque-speed transfer function can be obtained by multiplying the diagram of Fig. 4 by $1,5 p \lambda_{m g}$, where $p$ is the number of pole pairs and $\lambda_{m g}$ is the flux linkage due to the rotor permanent magnets.

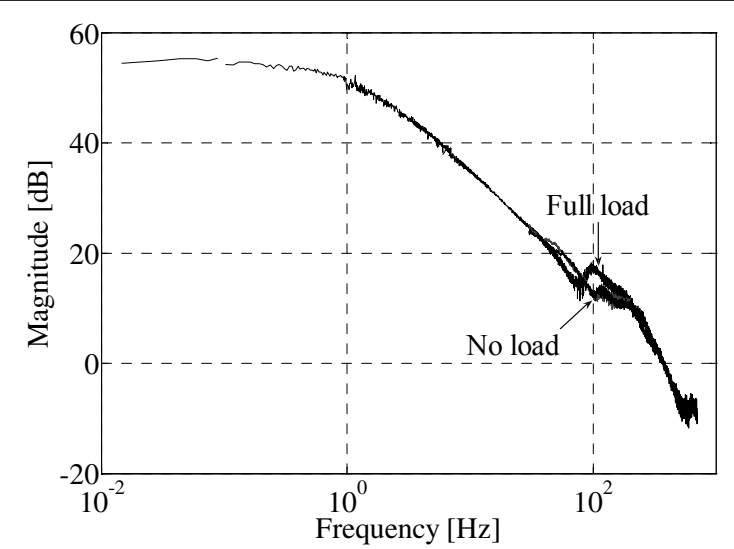

Fig.4 -Mechanical load Bode diagram: magnitude.

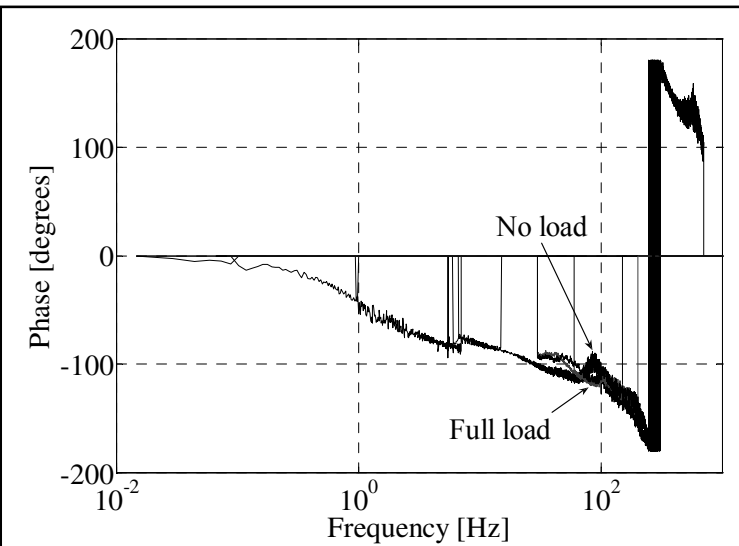

Fig.5 - Mechanical load Bode diagram: phase.

\section{Transfer function identification}

The Bode diagrams obtained in the previous paragraph have been analysed to obtain a mathematical description of the mechanical load in the Laplace domain form. To maintain a rather low implementation complexity, the structure of the system model was the result of a manual guess, aided by computer simulations. In case of real need, some more complex automatic procedures could be investigated.

The no-load transfer function that best fits for the experimental curves features two couples of complex poles and complex zeros, as follows:

$$
\begin{aligned}
& G M_{\text {noload }}=\frac{K_{m}}{s \tau_{m}+1} \cdot \frac{s^{2} / \omega_{z}^{2}+2 \xi_{z} / \omega_{z}+1}{s^{2} / \omega_{p 1}^{2}+2 \xi_{p 1} / \omega_{p 1}+1} \\
& \cdot \frac{\tau_{z} s+1}{s^{2} / \omega_{p 2}^{2}+2 \xi_{p 2} / \omega_{p 2}+1}
\end{aligned}
$$

where the parameters assume the following values: 


$$
\begin{array}{ccc}
K_{m}=520 & \tau_{m}=\frac{1}{2 \pi \cdot 1,05} & \tau_{z}=\frac{1}{2 \pi \cdot 135} \\
\omega_{z}=2 \pi 79,5 & \omega_{p 1}=2 \pi 89,5 & \omega_{p 2}=2 \pi 290 \\
\xi_{z}=0,175 & \xi_{p 1}=0,205 & \xi_{p 2}=0,5
\end{array}
$$

Similarly, the full-load transfer function has been modelled with three couples of complex poles and complex zeros, as reported hereafter:

$$
\begin{aligned}
G M_{\text {load }}= & \frac{K_{m}}{s \tau_{m}+1} \cdot \frac{s^{2} / \omega_{z 1}^{2}+2 \xi_{z 1} / \omega_{z 1}+1}{s^{2} / \omega_{p 1}^{2}+2 \xi_{p 1} / \omega_{p 1}+1} . \\
& \cdot \frac{s^{2} / \omega_{z 2}^{2}+2 \xi_{z 2} / \omega_{z 2}+1}{s^{2} / \omega_{p 2}^{2}+2 \xi_{p 2} / \omega_{p 2}+1} . \\
& \cdot \frac{s^{2} / \omega_{z 3}^{2}+2 \xi_{z 3} / \omega_{z 3}+1}{s^{2} / \omega_{p 3}^{2}+2 \xi_{p 3} / \omega_{p 3}+1}
\end{aligned}
$$

The numerical parameters, obtained by trial-and-error, were the following:

$$
\begin{array}{ccc}
K_{m}=520 & \tau_{m}=\frac{1}{2 \pi 1,05} & \omega_{z 1}=2 \pi 44 \\
\omega_{p 1}=2 \pi 50 & \omega_{z 2}=2 \pi 107 & \omega_{p 2}=2 \pi 115 \\
\omega_{z 3}=2 \pi 201 & \omega_{p 3}=2 \pi 215 & \xi_{z 1}=0,45 \\
\xi_{p 1}=0,43 & \xi_{z 2}=0,16 & \xi_{p 2}=0,19 \\
\xi_{z 3}=0,32 & \xi_{p 3}=0,18 &
\end{array}
$$

The comparison of the transfer function magnitude response with respect to measured data is reported in Fig. 6, for both the no-load and the full-load transfer function. The excellent match confirms the effectiveness of the used method.

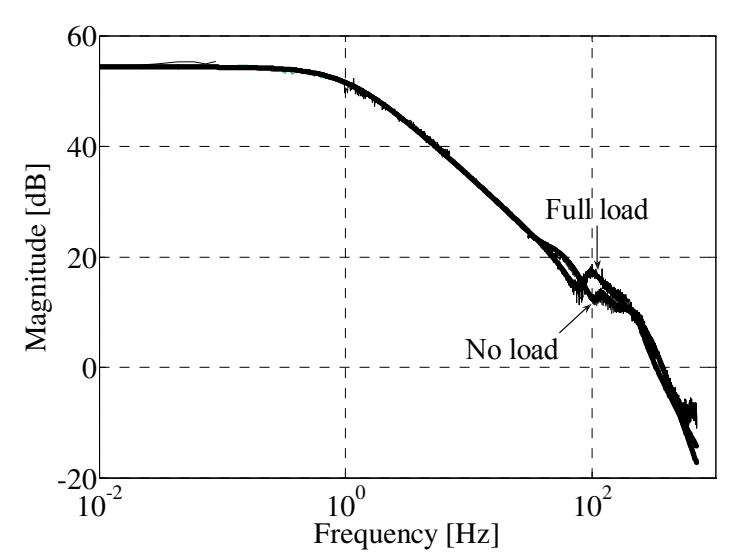

Fig.6 - Identified models vs. measured data.

\section{PMSM drive tuning}

Results from Section IV-E have been used to tune the speed regulator. Due to the small change of the identified transfer function from no-load to full-load conditions, the PI parameters has been fixed to constant values, obtaining almost the same performances for every condition. Fig. 7 and Fig. 8 report the simulation and experimental measurements of a speed step at no load, while Fig. 9 and Fig. 10 show the same step for a full-load condition. The identification of the load model and the estimation of its parameters were of great importance in speeding up the tuning.

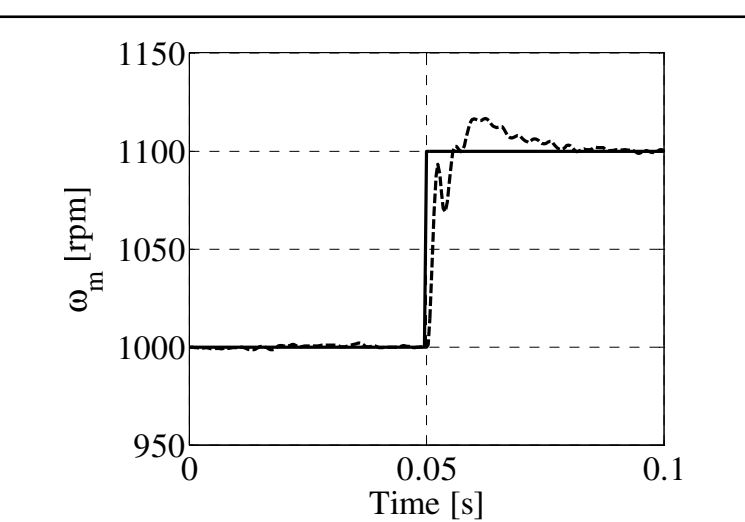

Fig. 7 - Speed step at no load, simulation results.

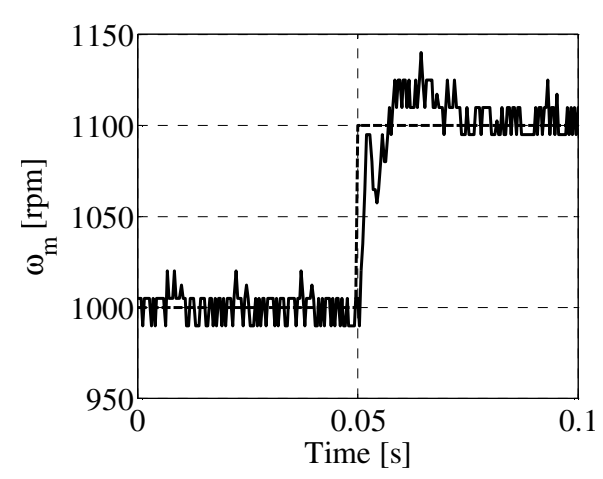

Fig. 8 - Speed step at no load, experimental results.

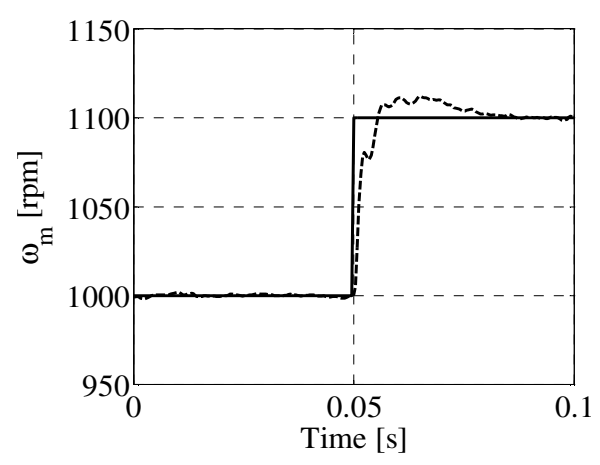

Fig.9 - Speed step at full load, simulation results. 


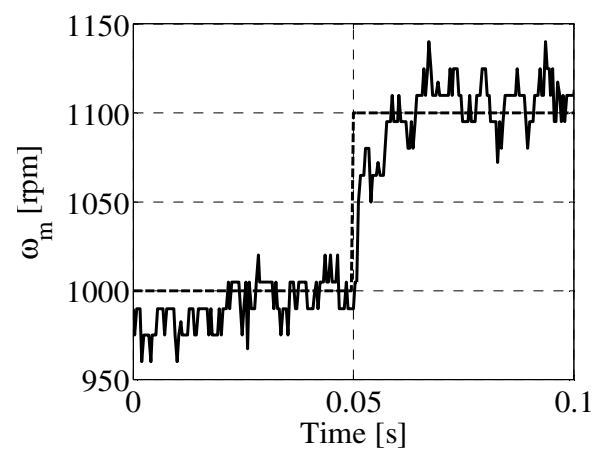

Fig.10 - Speed step at full load, experimental results.

\section{CONCLUSIONS}

The paper presented a method for the black-box model identification of a complex mechanical load. After recalling the mathematical basics of the proposed approach, the procedure has been fully tested on a case study, represented by a labelling machine. The PI speed controller of the PMSM drive has been tuned using the identified model, obtaining good performances that were also confirmed by the impressive matching between simulation and experimental results.

\section{ACKNOWLEDGEMENTS}

The Authors would like to thank ELTA Elettronica s.r.l., San Giovanni Lupatoto, Verona, Italy, and Etipack S.p.A., Cinisello Balsamo, Milano, Italy, for their generous support. A special thank to ing. A. Antonioli and M. Antonioli for the technical collaboration during the experimental stage of the project.

\section{REFERENCES}

[1]. C. C. Hang, K. J. Åström and W. K. Ho. Refinements of the Ziegler-Nichols tuning formula, IEE Proceedings D - Control Theory and Applications 138 (2) (1991). pp. 111-118.

[2]. P. Crnosija, R. Krishnan and T. Bjazic. Optimization of PM Brushless DC Motor Drive Speed Controller Using Modification of Ziegler-Nichols Methods Based on Bode Plots. Proceedings of the 12th International Power Electronics and Motion Control Conference (EPE-PEMC 2006), Portorož, Slovenia Aug. 30-Sep. 1 2006, p. 343-348.

[3]. S. Beineke, F. Schütte, H. Wertz, H. Grotstollen, Comparison of Parameter Identification Schemes for Self-Commissioning Drive Control of Nonlinear TwoMass Systems. Conference Record of the 32th Industry Applications Society Annual Meeting (IAS 1997), New Orleans, LA, USA, October 5-9 1997, pp. 493500 .

[4]. H. Wertz, S. Beineke, N. Frohleke, S. Bolognani, K. Unterkofler, M. Zigliotto, and M. Zordan. Computer aided commissioning of speed and position control for electrical drives with identification of mechanical load.
Conference Record of the $34^{\text {th }}$ Industry Applications Society Annual Meeting (IAS 1999), Phoenix, AZ, USA, October 3-7 1999, vol. 4, pp. 2372-2379.

[5]. F. Schütte, S. Beineke, H. Grotstollen. Knowledge Based Controller Selection for Electrical Drives with Imperfect Mechanic. Proceedings of the $24^{\text {th }}$ Annual Conference of the IEEE Industrial Electronic Society, Aachen, Germany, Aug. 31-Sept. 4 1998, pp. 14451450.

[6]. P. Mattavelli, L. Tubiana, M. Zigliotto. Simple control autotuning for PMSM drives based on feedback relay. Proceedings of the European Conference on Power Electronics and Applications (EPE 2005), Dresden, Germany, September 11-14 2005, pp. 10.

[7]. A. Chiuso. The role of Vector AutoRegressive Modeling in Predictor Based Subspace Identification. Automatica 43 (6) (2007), pp. 1034-1048.

[8]. A. Chiuso. On the Relation Between CCA and Predictor-Based Subspace Identification. IEEE Trans. Autom. Control 52 (10) (2007), pp. 1795-1812.

[9]. N. Benvenuto and G. Cherubini. Algorithms for Communications Systems and Their Applications. John Wiley and sons. 2002. ISBN 0470843896.

[10].B. R. Mahafza. Radar Systems Analysis and Design Using Matlab. Chapman \& Hall/CRC. 2000. ISBN 1584881828.

Luca Peretti received the M. Sc. Degree (cum laude) in Electronic Engineering from the University of Udine, Italy, and the Ph.D. in Mechatronics and Industrial Systems from the University of Padova, Italy. He is currently holding a post-doc position at the Department of Technique and Management of Industrial Systems, University of Padova, Vicenza. His research interests comprise sensorless control strategies and parameter estimation procedures for $\mathrm{AC}$ motor drives.

Mauro Zigliotto (M'88) is a native of Vicenza, Italy. He started his professional activity as $R \& D$ manager, developing microcontroller-based control systems for electric drives. From 1992 to 1999, he was a Senior Research Assistant in the Electric Drives Laboratory, University of Padova. Since 2000, for five years, he worked at the Department of Electrical, Management and Mechanical Engineering of the University of Udine, as Associate Professor of Electric Drives. In November, 2005 he joined the Department of Technique and Management of Industrial Systems, University of Padova, where he started the Electric Drives Laboratory. Advanced control strategies for ac motors are Prof. Zigliotto's main research interest, and he has published more than 100 papers in this area. He has been serving International Conferences as member of the Steering or Technical Committees. At present he is the secretary of the IEEE IAS-IES-PELS North Italy Joint Chapter. 